\title{
Ion-Pair Extraction and Flame Atomic Absorptiometric Determination of Copper in Standard Reference Materials
}

\author{
Isao KojIma, ${ }^{\dagger}$ Mamoru Ito, Tomoyuki Mizuno, Akihiro Matsumori, and Yoko Asai \\ Laboratory of Analytical Chemistry, Department of Applied Chemistry, Nagoya Institute of Technology, \\ Gokiso, Showa, Nagoya 466-8555, Japan
}

\begin{abstract}
The ion-pair extraction of univalent copper with 3,6-dithiaoctane in the presence of each of 15 univalent anions into a small volume of chloroform at a $\mathrm{pH}$ of less than 7 was studied. Of these anions, picric acid, Bromothymol Blue, and cobalt(III)-dihydroxyazobenzene complex gave a quantitative extraction of copper (>99\%) and Bromocresol Green, EO, PO, and Co-5-Br-PAPS complex a constant, but a slightly lower, extraction (85-95\%) at their corresponding $\mathrm{pH}$ ranges, respectively. The direct nebulization of $50 \mu \mathrm{L}$ of the chloroform extract into a fuel-lean air-acetylene flame gave a sensitive signal height without any background correction from chloroform. The present method was successfully applied to the determination of copper in three standard botanical, one standard zoological, and four standard geological reference materials.
\end{abstract}

(Received June 22, 2000; Accepted August 28, 2000)

By a one-drop flame atomic absorption spectrometry (FAAS) with the direct nebulization of $50 \mu \mathrm{L}$ of the organic extract into a fuel-lean air-acetylene flame, some elements have been sensitively determined without any background correction. With the direct nebulization of the organic extract, tedious and time-consuming pre-procedures before FAAS determination, e.g., the back extraction or the evaporation of the organic solvent and the mineralization of the residue, have been avoided. With this technique, copper in a standard reference sample (NIES-CRM No. 9, Sargasso) was well determined, after the extraction of copper-dithizonate into carbon tetrachloride. ${ }^{2}$

Of many organic reagents, cyclic and/or acyclic organic reagents containing oxygen and/or nitrogen atoms as coordinating atoms have been used in the field of analytical chemistry of metals, especially alkali and alkaline earth elements. Recently, it has been reported that acyclic thioethers, obtained by replacing the oxygen atoms with sulfur atoms, have a great affinity for soft metals and extract these metal ions selectively as an ion-pair complex in the presence of a bulky univalent anion. ${ }^{3,4}$ In previous studies, the combined use of the selective extraction of the ion-pair silver complex into a small volume of chloroform in the presence of picrate or perchlorate ion and the direct nebulization of $50 \mu \mathrm{L}$ of the chloroform extract into a fuel-lean air-acetylene flame was well applied to the determination of silver in highly pure copper and lead metals ${ }^{5}$ and in nine biological standard reference materials. ${ }^{6}$

The present paper describes the possibility of the selective extraction of copper(I) with a commercially available 3,6dithiaoctane (8-2S) at a $\mathrm{pH}$ of less than 7 into a small volume of chloroform in the presence of each anion of 15 univalent anions and a one-drop FAAS determination of copper in standard biological and geological samples. The standard samples used

† To whom correspondence should be addressed.

E-mail: kojima@ach.nitech.ac.jp are Rice Flour (NIES-CRM, No. 10), Mussel (NIES-CRM, No. 6), and Chlorella (NIES-CRM, No. 3) from National Institute of Environmental Studies, Citrus Leaves (NIST-SRM, No. 1572) from National Institute of Standards and Technology, JB-1, JB1a and JA-1 from Geological Survey of Japan (GSJ) and GSP-1 from United States Geological Survey (USGS).

\section{Experimental}

\section{Apparatus}

A flame atomic absorption spectrometer with a 100-mm burner head (Seiko Model SAS-727) was used for copper determinations with a one-drop technique using a fuel-lean air-acetylene flame under the optimum operating conditions: flow-rate of acetylene, $3.5 \mathrm{~L} \mathrm{~min}^{-1}(49 \mathrm{kPa})$; flow-rate of air, 17 $\mathrm{L} \min ^{-1}(245 \mathrm{kPa})$; observation height, $13.5 \mathrm{~mm}$ above the burner head and wavelength, $324.8 \mathrm{~nm}$. The sample solution of $50 \mu \mathrm{L}$ was injected with a micropipette (Gilson P-200) into the PTFE funnel coupled directly to the nebulizer needle. The signal intensity was recorded on a strip-chart recorder (Nippon Denshi Kagaku, Unicorder U-228). The $\mathrm{pH}$ of the aqueous solution was measured with a Radiometer Type PHM-22 pH meter (Copenhagen, Denmark) with a combined electrode.

\section{Chemicals}

A copper stock solution ( $2 \mathrm{mg} \mathrm{g}^{-1}$ in $0.5 \mathrm{~mol} \mathrm{dm}^{-3}$ nitric acid) was prepared by dissolving copper metal of $99.999 \%$ purity (Mitsuwa Chemicals, Osaka) in nitric acid directly in a PTFE bottle of $120 \mathrm{~cm}^{3}$ capacity, followed by dilution by mass with water. Working copper standard solutions of the chloroform extract were prepared by extracting the copper(II)-APDC complex or ion-pair copper(I) complex into chloroform. Chloroform of analytical reagent grade was used without further purification. Commercially available $8-2 \mathrm{~S}$ of analytical reagent 
grade (Wako Pure Chemicals, Osaka) was used by dissolving in chloroform $\left(0.05 \mathrm{~mol} \mathrm{dm}^{-3}\right)$. Aqueous ammonium pyrrolidin-1yldithiocarbamate (APDC, Nacalai Tesque, Osaka) solution $(1 \% \mathrm{~m} / \mathrm{m})$ was prepared by dissolving in water. Ethyl Orange (EO, $3 \times 10^{-3} \mathrm{~mol} \mathrm{dm}^{-3}$ ), sodium tetraphenylborate (TPB, 10-2 mol $\mathrm{dm}^{-3}$ ), Bromocresol Green (BCG, 10-3 mol $\mathrm{dm}^{-3}$ ), Bromothymol Blue (BTB, $\left.10^{-3} \mathrm{~mol} \mathrm{dm}^{-3}\right)$ and picric acid $\left(10^{-2}\right.$ mol $\mathrm{dm}^{-3}$ ) were from Wako. $O$-o'-Dihydroxyazobenzene (DHAB, $\left.10^{-3} \mathrm{~mol} \mathrm{dm}^{-3}\right)$ and disodium 2-[(5-bromo-2pyridyl)azo]-5-[ $N$-propyl- $N$-(3-sulfopropyl)amino]phenolate (5Br-PAPS, $10^{-3} \mathrm{~mol} \mathrm{dm}^{-3}$ ) were from Dojindo Laboratories (Kumamoto, Japan). Propyl Orange (PO, $10^{-3} \mathrm{~mol} \mathrm{dm}^{-3}$ ) donated from Prof. Motomizu of Okayama University was used without further purification. These anion-forming substances were dissolved in water or in an appropriate volume of a 0.1 mol dm${ }^{-3}$ sodium hydroxide solution. The solutions $\left(3.3 \times 10^{-3}\right.$ mol $\mathrm{dm}^{-3}$ ) of the Co-DHAB and Co-5-Br-PAPS complexes were prepared by mixing the cobalt and DHAB or 5-Br-PAPS solutions in a 1:2 molar ratio. The acids, sodium acetate $(2 \mathrm{~mol}$

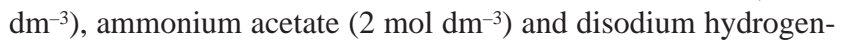
phosphate $\left(1 \mathrm{~mol} \mathrm{dm}^{-3}\right)$ of analytical reagent grade (Wako, Osaka) were used to control the extraction $\mathrm{pH}$. L-Ascorbic acid $(1 \% \mathrm{~m} / \mathrm{v})$ of analytical grade and hydroxylammonium chloride for metal analysis from Wako were used as a reducing agent. Deionized Milli-Q water of $18.2 \mathrm{M} \mathrm{ohm} \mathrm{cm}^{-1}$ was used throughout.

\section{Decomposition of rock samples}

A sample of $200 \mathrm{mg}$ was taken in a PTFE vessel of $15 \mathrm{~cm}^{3}$ capacity with $4 \mathrm{~cm}^{3}$ of nitric and $4 \mathrm{~cm}^{3}$ of hydrofluoric acids. After sealing by hand, the vessel was left standing overnight at room temperature, and then evaporated to dryness on a hot-plate and again with $2 \mathrm{~cm}^{3}$ of nitric acid. Finally, the residue was dissolved in $10 \mathrm{~cm}^{3}$ of a $0.05 \mathrm{~mol} \mathrm{dm}-3$ sulfuric acid and the total mass of the sample solution was weighed. An aliquot of the thus-prepared solution was used for the determination of copper.

\section{Decomposition of rice flours}

A sample of $400 \mathrm{mg}$, dried at $85^{\circ} \mathrm{C}$ for $4 \mathrm{~h}$ just before use, was taken in a PTFE vessel of $15 \mathrm{~cm}^{3}$ capacity with $5 \mathrm{~cm}^{3}$ of nitric, $0.6 \mathrm{~cm}^{3}$ of perchloric, $0.6 \mathrm{~cm}^{3}$ of hydrochloric, and $0.6 \mathrm{~cm}^{3}$ of hydrofluoric acids. After sealing by hand, the vessel was left standing overnight at room temperature, and then the evolving gas was expelled by gentle heating on a hot-plate for a while. The vessel was placed in an outer PTFE vessel of $50 \mathrm{~cm}^{3}$ capacity containing $3 \mathrm{~cm}^{3}$ of $1 \mathrm{~mol} \mathrm{dm}^{-3} \mathrm{NaOH}$ solution with the polypropylene jacket. The sample in this vessel was decomposed by microwave-assisted heating, as was done in a previous study. ${ }^{7}$ The digest in the inner vessel was completely evaporated to dryness. The dry residue was dissolved in 2.0 $\mathrm{cm}^{3}$ of a $0.05 \mathrm{~mol} \mathrm{dm}^{-3}$ sulfuric acid in the same vessel. The total mass of the sample solution was weighed. The thusprepared sample solution was used for the determination of copper.

\section{Decomposition of other biological samples}

A sample of $100-150 \mathrm{mg}$, dried at $85^{\circ} \mathrm{C}$ for $4 \mathrm{~h}$ just before use, was taken in a PTFE vessel of $15 \mathrm{~cm}^{3}$ capacity with $3 \mathrm{~cm}^{3}$ of nitric, $0.6 \mathrm{~cm}^{3}$ of perchloric, $0.3 \mathrm{~cm}^{3}$ of hydrochloric, and 0.3 $\mathrm{cm}^{3}$ of hydrofluoric acids. After treating these, as described just above, the residue was dissolved in $1.0-3.0 \mathrm{~cm}^{3}$ of a $0.05 \mathrm{~mol}$ $\mathrm{dm}^{-3}$ sulfuric acid in the same vessel, of which the volume corresponds to the respective copper contents, and the total mass of the sample solution was weighed. The thus-prepared sample solution was used for the determination of copper.

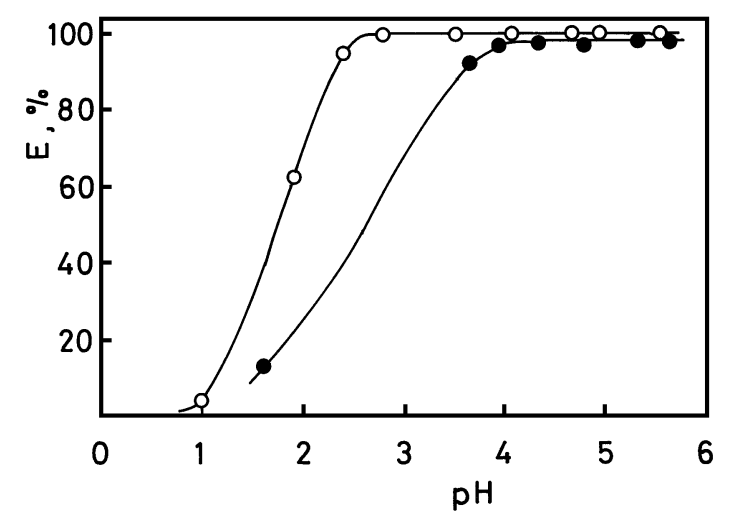

Fig. 1 Effect of $\mathrm{pH}$ on copper extraction. Reducing agent: open circle, ascorbic acid; filled circle, hydroxylamine. Anion: Co(III)DHAB complex.

\section{Extraction procedure}

a) Into a $10-\mathrm{cm}^{3}$ stoppered glass test-tube, $0-1.0 \mathrm{~cm}^{3}$ of the standard solution of $1.0 \mu \mathrm{g} \mathrm{g}^{-1}$ (weighed), $1.0 \mathrm{~cm}^{3}$ of the APDC solution, $4-3 \mathrm{~cm}^{3}$ of water, and $1.0 \mathrm{~cm}^{3}$ of chloroform (weighed) were placed. After shaking for $20 \mathrm{~s}$ (three times) by hand, phase separation was carried out by centrifuging at $3000 \mathrm{rpm}$. The calibration curve obtained with these chloroform extracts was used for estimating the percent extraction of the ion-pair copper complex in the presence of each univalent anion. The signal height on the strip-chart recorder was used for the copper determination.

b) Into a $10-\mathrm{cm}^{3}$ stoppered glass test-tube, $1.0 \mathrm{~cm}^{3}$ of the sample solution (weighed) or $1.0-0 \mathrm{~cm}^{3}$ of the standard copper solution (weighed), $0.5 \mathrm{~cm}^{3}$ of an ammonium acetate or a sodium acetate, $1.0 \mathrm{~cm}^{3}$ of a reducing agent solution, $0.5 \mathrm{~cm}^{3}$ of the anion solution, 2 (or $3-2$ ) $\mathrm{cm}^{3}$ of water, and 0.5 (or 1.0) $\mathrm{cm}^{3}$ of the $8-2 \mathrm{~S}$ chloroform solution (weighed) were placed. After shaking for $20 \mathrm{~s}$ (three times) by hand, phase separation was carried out by centrifuging at $3000 \mathrm{rpm}$. The chloroform extract was used for copper measurements. The copper content in the extracts can be found by consulting the calibration curve obtained with the similarly treated standard chloroform extracts.

\section{Results and Discussion}

\section{Extraction behavior of copper with 8-2S}

The extraction behavior of copper from the aqueous solution in the presence of each of the univalent organic anions was examined in the $\mathrm{pH}$ range less than 7 . The results obtained with ascorbic acid as a reducing agent were given in a previous paper. ${ }^{8}$ Taking into account these results, the extraction of copper was also studied with hydroxylamine as a reducing agent and with five organic anions. The results obtained with the CoDHAB complex as a counter anion are shown in Fig. 1, as a typical example. As is well-known, copper was extracted at a slightly higher $\mathrm{pH}$ region in the presence of hydroxylamine, compared with the case of ascorbic acid, because of the decrease in the reducing ability of hydroxylamine along with an increase in the hydrogen-ion concentration. Irrespective of the reducing agent, the constant and high extraction of the ion-pair copper complex in the presence of each organic anion was obtained at the corresponding optimal $\mathrm{pH}$ region. These results are summarized in Table 1. The extraction behavior of copper in the presence of inorganic univalent anions was also examined 
Table 1 Optimal extraction $\mathrm{pH}$ range of ion-pair copper complexes

\begin{tabular}{lccccc}
\hline \multicolumn{1}{c}{ Anion } & $\begin{array}{c}\text { Concn./ } \\
10^{-5} \mathrm{~mol} \mathrm{dm}^{-3}\end{array}$ & $\mathrm{pH}$ range & $E, \%$ & $\mathrm{pH}$ range & $E, \%$ \\
\hline Picrate & 10 & $3-6$ & $>99$ & $4-5$ & 96 \\
TPB & 10 & $3-5.5$ & 75 & - & - \\
BCG & 10 & $3-4.5$ & 95 & $4.3-5$ & 93 \\
BTB & 5 & $2-7$ & $>99$ & $4-7$ & $>99$ \\
Co-5-Br-PAPS & 4 & $3-4.5$ & 85 & - & - \\
5-Br-PAPS & 5 & $3-3.5$ & 75 & - & - \\
Co-DHAB & 8 & $3-4.5$ & $>99$ & $4.4-5.5$ & 96 \\
EO & 30 & $4-6$ & 90 & $5-5.5$ & 90 \\
PO & 4 & $3.6-6$ & 95 & - & - \\
Reductant & - & Ascorbic acid & $\mathrm{NH}_{2} \mathrm{OH}$ \\
\hline
\end{tabular}

Table 2 Analytical results for copper in rice flour $\left(\mu \mathrm{g} \mathrm{g}^{-1}\right)$

\begin{tabular}{ccccccc}
\hline Sample & $\begin{array}{c}\text { Co-5- } \\
\text { Br-PAPS }\end{array}$ & BTB & BCG & $\begin{array}{c}\text { Co- } \\
\text { DHAB }\end{array}$ & EO & Certified $^{9}$ \\
\hline a & 3.5 & 3.3 & 3.3 & 3.3 & 3.2 & $3.5 \pm 0.3$ \\
b & 3.2 & 3.1 & 3.3 & 3.3 & 2.7 & $3.3 \pm 0.2$ \\
c & 4.2 & 4.1 & 3.9 & 4.2 & 4.1 & $4.1 \pm 0.3$ \\
\hline
\end{tabular}

Sample: NIES-CRM No. 10-a, b and c.

a, low-level Cd; b, medium-level Cd; c, high-level Cd.

using only ascorbic acid. However, the extraction of copper was unsatisfactory in the presence of each of the inorganic univalent anions; also, the constant extraction was not obtained at any $\mathrm{pH}$ region.

\section{Effect of anion concentration}

The extraction of the ion-pair copper complex depends on the kind of anions and their concentration, but not on the kind of reducing agents at the optimal extraction $\mathrm{pH}$ range. This is easily recognized from the data in Table 1 . With organic anion concentration ranges of $(4-20) \times 10^{-5} \mathrm{~mol} \mathrm{dm}^{-3}$, the quantitative extraction of copper $(>99 \%)$ was attained for picrate ion, BTB and Co-DHAB complex with ascorbic acid as a reducing agent and for BTB with hydroxylamine at the optimal extraction $\mathrm{pH}$ ranges of $4.5-5$, respectively. But with BCG, EO, PO, and Co-5-Br-PAPS complex, the constant and a slightly lower percent extraction $(85-95 \%)$ was obtained at their optimal extraction $\mathrm{pH}$ ranges with each reducing agent, respectively. Even with the organic anions giving a constant, but slightly lower, percent extraction of copper, copper will be determined by using a similarly treated calibration curve. On the other hand, about $75 \%$ of copper(I) and $20 \%$ of copper(II) were extracted with TPB, though copper(II) was not extracted with the other anions studied here.

\section{Application to standard samples}

The obtained calibration curve gave a straight line with correlation coefficient of more than 0.998 . The reproducibility was also $1.5 \%$ at $0.5 \mu \mathrm{g} \mathrm{cm}^{-3}$ of copper in the chloroform extract. Also, the sensitivity of the present one-drop FAAS is 1.5-times larger than that obtained by nebulizing an aqueous solution. On the basis of these facts, the present method was applied to the determination of copper in standard biological and geological samples from National Institute for Environmental Studies (NIES-CRM), National Institute of Standards and Technology (NIST-SRM), Geological Survey of Japan (GSJ) and United States Geological Survey (USGS). The
Table 3 Analytical results for copper in standard samples $\left(\mu \mathrm{g} \mathrm{g}^{-1}\right)$

\begin{tabular}{lrc}
\hline \multicolumn{1}{c}{ Sample } & Found & Certified \\
\hline Mussel (NIES-CRM, No. 6) & 4.6 & $4.9 \pm 0.3^{9}$ \\
Chlorella (NIES-CRM, No. 3) & 3.5 & $3.5 \pm 0.3^{9}$ \\
Citrus Leaves (NIST-SRM,1572) & 16.2 & $16.5 \pm 1.0^{10}$ \\
\hline
\end{tabular}

Anion used: BTB.

Table 4 Analytical results for copper in standard rock samples

\begin{tabular}{lcccccc}
\hline & \multicolumn{6}{c}{ Copper found $/ \mu \mathrm{g} \mathrm{g}^{-1}$} \\
& BTB & BCG & Co(DHAB) & Picrate & EO & Reported $^{11}$ \\
\cline { 2 - 7 } JA-1 & 40.5 & 40.7 & 40.5 & 40.8 & 41.9 & 42.2 \\
& 40.7 & 40.5 & 40.4 & 41.4 & 42.4 & \\
& - & - & - & - & 43.8 & \\
JB-1 & 53.2 & 52.3 & 51.8 & 51.7 & - & 56.3 \\
JB-1a & 52.3 & 55.1 & 50.7 & 57.9 & 52.9 & 55.5 \\
& - & - & - & - & 55.5 & \\
GSP-1 & 30.9 & 30.7 & - & - & 54.3 & \\
& 33.2 & 31.5 & 30.8 & 31.7 & 32.7 & \\
\hline
\end{tabular}

analytical results are given in Tables $2-4$ along with their certified or reported values. The analytical results agreed well with the certified or reported values, and are also very reproducible.

In conclusion, univalent copper was efficiently extracted into chloroform as an ion-pair complex in the presence of univalent organic anion. Copper in the samples was also well-determined by a one-drop FAAS, irrespective of the use of sodium and ammonium acetates as a buffer component, and also of the use of ascorbic acid and hydroxylamine as a reducing agent of copper. On the other hand, nothing about the spectrophotometric results is given here, in spite of the use of the colored organic anions. With the use of some of the colored organic anions studied in this paper, copper was not determined spectrophotometrically, owing to positive interference from the coexisting component in the extraction. To overcome these difficulties, a spectrophotometric study is now in progress and will be published elsewhere along with applications to practical samples.

\section{Acknowledgements}

The authors wish to deeply thank Prof. Motomizu of Okayama University for the donation of highly pure Propyl and Butyl Oranges.

\section{References}

1. I. Kojima, K. Inagaki, and S. Kondo, J. Anal. At. Spectrom., 1994, 9, 1161 and cited therein.

2. I. Kojima, N. Nakashima, H. Isoyama, T. Uchida, and C. Iida, J. Anal. At. Spectrom., 1988, 3, 583.

3. A. Ohki, M. Takagi, and K. Ueno, Anal. Chim. Acta, 1984, 159, 245.

4. K. Chayama, N. Koyama, H. Tsuji, and E. Sekido, Bunseki Kagaku, 1998, 47, 993. 
5. I. Kojima and A Takayanagi, J. Anal. At. Spectrom., 1966, $11,607$.

6. I. Kojima and M. Katsuzaki, Anal. Sci., 1997, 13, 1021.

7. I. Kojima, A. Kato, and C. Iida, Anal. Chim. Acta, 1992, 264,101 .

8. I. Kojima, M. Ito, and T. Mizuno, in "Proceedings of the ISEC' 99", 1999, Barcelona, in press.

9. K. Okamoto, Sci. Total Environ., 1991, 107, 29.
10. K. S. Gladney, B. T. O'Malley, I. Roelandts, and T. E. Gills, "Standard Reference Materials: Compilation of Elemental Concentration Data for NBS Clinical, Biological, Geological and Environmental Standard Reference Materials", 1987, National Bureau of Standards, Spec. Publ., 260 - 111.

11. K. Govindaraju, Geochemical Newslet., 1989, Vol. 13, Special Issue. 